\title{
ON MODELLING THE DRYING OF POROUS MATERIALS: ANALYTICAL SOLUTIONS TO COUPLED PARTIAL DIFFERENTIAL EQUATIONS GOVERNING HEAT AND MOISTURE TRANSFER
}

DON KULASIRI AND IAN WOODHEAD

Received 10 August 2004 and in revised form 2 December 2004

Luikov's theory of heat and mass transfer provides a framework to model drying porous materials. Coupled partial differential equations governing the moisture and heat transfer can be solved using numerical techniques, and in this paper we solve them analytically in a setting suitable for industrial drying situations. We discuss the nature of the solutions using the physical properties of Pinus radiata. It is shown that the temperature gradients play a significant role in deciding the moisture profiles within the material when thickness is large and that models based only on moisture potential gradients may not be sufficient to explain the drying phenomena in moist porous materials.

\section{Introduction}

Porous materials such as wood, grains, fruit, and dairy products have microscopic capillaries and pores which cause a mixture of transfer mechanisms to occur simultaneously when subjected to heating or cooling. Transfer of noncondensable gases, vapours, and liquids occurs in porous bodies; inert gases and vapour transfer can take place by molecular means in the form of diffusion and by molar means as a filtration motion of the steam-gas mixture under a pressure gradient. Transfer of liquids can occur by means of diffusion, capillary absorption, and filtration motion in the porous medium arising from the hydrostatic pressure gradient. The complex interactions of various phenomena occurring within a material undergoing heating and cooling make modelling the transient moisture and temperature within the body a difficult task. Empirical models dealing with the drying of porous materials ignore temperature variation within the material and formulate the models in terms of a measure of the moisture content of the body and the equilibrium moisture content of the material $[7,8,15]$.

Temperature variations are introduced to the models by relating the coefficients of the models to external temperature and humidity. These empirical relationships give satisfactory results in many industrial situations. In modelling drying, the most widely used mass transport model is Fick's second law [4] and analytical solutions can be obtained for isotropic and anisotropic conditions $[2,4]$. Similarly heat conduction equation can be solved analytically [4] and in many cases it is sufficient to solve the governing partial 
differential equations separately without paying attention to the coupling effects, especially when the drying rates are small (see, e.g., [3]). However, one would expect that these coupled heat and mass transfer in porous bodies could be expressed mathematically and hence a mechanistic model can be developed. To this end, we make use of the theory developed by Luikov [9] to formulate a model of heat and mass transfer within the material.

Luikov showed the importance of the temperature gradient for moisture migration in capillary-porous bodies [9]. He developed a system of coupled PDEs using the thermodynamics of irreversible processes. Fulford [6] surveyed work on the drying of solids by Luikov and his colleagues and no attempt will be made here to describe Luikov's rigorous theoretical development. Luikov assumed that both vapour and liquid diffusion are driven by both the total concentration gradient and the temperature gradient. He assumed that molecular and molar transfer of air, vapour, and water occurred simultaneously within the porous body. Luikov stated that these coupled equations could not be solved and therefore have to be simplified [9]. When the coupling effects are important, one can solve the coupled equations using numerical methods such as the finite element method for a given situation, the accepted practise among scientists and engineers.

\subsection{Numerical modelling of coupled (conjugate) heat and mass transfer in drying.}

Thomas et al. [14] used the coupled two-dimensional PDEs based on Luikov's theory to develop a fully nonlinear finite element formulation to solve a problem of kiln drying timber having a cross section of $200 \mathrm{~mm} \times 50 \mathrm{~mm}$. Then they simplified the numerical problem using linear finite element formulation for the same cross section of timber and concluded that the use of fully nonlinear formulation was not justified because there was no difference in results from both formulations. Constant stationary initial conditions through the wood were assumed (initial temperature $=10^{\circ} \mathrm{C}$, and the initial moisture content $=0.30$ (decimal, dry basis) ) and constant boundary conditions were also used: surface temperature and moisture at the surface $60^{\circ} \mathrm{C}$ and 0.12 (decimal, dry basis), respectively. Contextually similar study in solving a conjugate heat and mass transfer model was given by Olivera and Haghighi [11]. By using adaptive finite element techniques and incorporating the laminar flow over a solid piece of wood, they solved the governing coupled PDEs for the drying of wood.

Murugesan et al. [10] developed a theoretical model of brick drying in two dimensions based on liquid, vapour, and energy balances as well as on Darcy's law for capillary liquid mass flux and Fick's law for diffusive mass flux. The flow field over the solid was modelled by the Navier-Stokes equations. There are a total of five governing equations including a pair of conjugate PDEs modelling the behaviour of temperature and moisture variables within the body. These conjugate models however were derived from mass and energy conservation laws, and the scientific rationale on which these derivations are based is different from that of Luikov [9]. The governing equations were solved using the finite element method with triangular elements over a rectangular flow domain within which a solid (brick) rectangular domain was embedded at the centre of the flow domain. In contrast to the much involved numerical modelling, a simple graphical method was proposed by Sahin and Dincer [13] to determine the moisture diffusivity and moisture 
transfer coefficient for solid products. The method is based on the analytical solution of one-dimensional Fickian equation in a dimensionless form for the moisture transfer for porous bodies without taking the coupling effects into account. Dolinskiy et al. [5] studied the problem of heat and mass exchange between a heat-transfer agent and continuous material pulled through it. The PDEs formulated were solved numerically by a difference technique which included an implicit difference scheme along with a tridiagonal matrix algorithm. The study is based on coupled PDEs very similar to Luikov's equations but series expansions were assumed a priori for the heat and mass fluxes, and the coefficients of which were calculated iteratively.

The main advantages of numerical solutions of the conjugate PDEs are (1) any geometry with irregular boundaries can be used as the spatial domains of the problems, and (2) tractable models can be developed for real industrial situations. However, there are many instances where analytical solutions of simplified PDEs could be useful in understanding the significance of crucial parameters, especially the coupling effects, and in extending the system behaviour to untested regimes. The simplicity of solutions would be insightful in understanding the physics of the problem at hand, and the solutions would be simpler to implement on computer. Further, analytical solutions of PDEs are used to validate numerical solution procedures and establish their accuracy [12]. The analytical solutions for simplified boundary and initial conditions can be used to gain insight into drying behaviour of porous materials and also to develop empirical relationships in industrial situations, as most empirical drying models have an underlying theoretical basis.

1.2. Purpose of the study. The main purpose of this study is to provide a brief summary of the development of a simplified set of Luikov's equations governing heat and mass transfer in porous media, and solve them analytically to explore the behaviour of the model in relation to the coupling parameters and material properties.

\section{Modelling heat and mass transfer}

Consider a system consisting of a porous body and a bound substance, which can be in the form of a liquid, vapour, or inert gas under positive temperature regimes but can be in the form of a solid (ice), a subcooled liquid or vapour, or a gas. Luikov developed a theory of mass and heat transfer for what he called capillary-porous bodies using the principles of irreversible thermodynamics [9]. In this paper, we consider cellular solids, materials consisting of cells, to be of this category, although there are significant differences between porous solids such as ceramics and cellular solids such as softwood. We modify Luikov's equations for positive temperature regimes to more realistically represented drying situations. Luikov and his coworkers showed that the thermal and moisture potential gradients within a capillary-porous body cause vapour diffusion and transfer of liquid water. A porous body above freezing temperatures can be considered a moist disperse system consisting of four different components: a dry porous skeleton (solid), water vapour, liquid water, and air within capillaries and pores. Using the subscript $i$ to denote the $i$ th component ( $i=0$ for bone-dry solid, $i=1$ for water vapour, $i=2$ for liquid water, and $i=3$ for air), and after neglecting the water vapour and air masses, the mass transfer 
of bound water can be modelled by the following conservation equation assuming that the moist material can be regarded as a continuum [9]:

$$
\frac{\partial\left(\rho_{0} m\right)}{\partial t}=-\nabla \cdot\left(J_{1}+J_{2}\right)
$$

where $m$ is the moisture content of the body (dry basis), $\rho_{0}$ is the density of the bone-dry solid, $J_{i}$ denotes the mass flux of component $i$, and $t$ is time. The heat transfer within the material can be modelled by the energy conservation equation

$$
c \rho_{0} \frac{\partial T}{\partial t}=-\nabla \cdot q-\left(H_{1} I_{1}+H_{2} I_{2}\right)
$$

where $c$ is the weighted specific heat of the moist solid referred to as the unit mass of the dry solid, $T$ is the temperature of the dispersed system, $q$ is the total heat flux, $H_{i}$ is the enthalpy per unit mass, and $I_{i}$ denotes the mass formation or disappearance rate during the phase changes.

Solutions to (2.1) and (2.2) with appropriate boundary and initial conditions give the moisture and temperature profiles within the material. However, before solving (2.1) and (2.2) with appropriate boundary and initial conditions, the mass flux $J_{i}$ and the heat flux $q$ should be expressed in terms of driving forces (moisture potentials and temperature gradients). Using the thermodynamics of irreversible processes and experiments, Luikov [9] proved the existence of two driving forces for mass transfer: the moisture concentration gradient and the temperature gradient for each of the mass fluxes. This means that the vapour diffusion and transfer of liquid water within the material can occur due to moisture concentration gradient and/or temperature gradient (thermodiffusion effect). Hence, $J_{1}$ and $J_{2}$ in (2.1) can be replaced by

$$
\begin{aligned}
& J_{1}=-a_{m 1} \rho_{0} \nabla m-a_{m 1}^{T} \rho_{0} \nabla T, \\
& J_{2}=-a_{m 2} \rho_{0} \nabla m-a_{m 2}^{T} \rho_{0} \nabla T,
\end{aligned}
$$

where $a_{m 1}, a_{m 2}$ are the effective diffusion coefficients for water vapour and liquid, respectively, and $a_{m 1}^{T}$ and $a_{m 2}^{T}$ are the corresponding thermal moisture diffusion coefficients. Substituting (2.3a) and (2.3b) into (2.1),

$$
\frac{\partial m}{\partial t}=\nabla \cdot\left[a_{m} \nabla m+a_{m} \delta \nabla T\right]
$$

where $a_{m}$ is the total diffusion coefficient, $a_{m}=a_{m 1}+a_{m 2}$, and $\delta$ is the thermogradient coefficient

$$
\delta=\frac{\left(a_{m 1}^{T}+a_{m 2}^{T}\right)}{\left(a_{m 1}+a_{m 2}\right)} .
$$


The thermo gradient coefficient is a measure of relative significance of the mass transfer due to the thermal gradient. The total heat flux $q$ in (2.2) is replaced by

$$
q=-k \nabla T
$$

where $k$ is the thermal conductivity of the moist material and the $\left(H_{1} I_{1}+H_{2} I_{2}\right)$ term is replaced by

$$
H_{1} I_{1}+H_{2} I_{2}=-R \varepsilon \frac{\partial m}{\partial t}
$$

where $R$ is the specific enthalpy of phase change and $\varepsilon$ is the phase change coefficient [6]. The phase change coefficient $\varepsilon$ varies from 0 to 1 as the vapour diffusion increases relative to liquid transfer during drying. After substituting (2.6) and (2.7) into (2.2),

$$
c \rho_{0} \frac{\partial T}{\partial t}=\nabla \cdot(k \nabla T)+R \varepsilon \frac{\partial m}{\partial t}
$$

Luikov compiled experimental values of $a_{m}, \delta, \rho_{0}, k$, and $c$ for a large number of porous materials. For example, values of $a_{m}$ for Pinus radiata wood varies from $1.0 \times 10^{-6} \mathrm{~m}^{2} / \mathrm{h}$ to $6.2 \times 10^{-6} \mathrm{~m}^{2} / \mathrm{h}$ and $\delta$ varies from $0.6 \times 10^{-2}$ to $2.0 \times 10^{-2}$ for the same temperature.

For all practical purposes, the heat and mass transfer in a porous body can be simplified into (2.4) and (2.8). The system of equations given by (2.4) and (2.8) is coupled, and nonlinear PDEs, whose behaviour can be investigated analytically.

Equations (2.4) and (2.8) can further be simplified by assuming constant values for the parameters $a_{m}, c, \rho_{0}, k$, and $\delta$ along the spatial dimension. Therefore, we seek to solve the following system of equations:

$$
\begin{gathered}
\frac{\partial m}{\partial t}=a_{m} \nabla^{2} m+a_{m} \delta \nabla^{2} T, \\
c \rho_{0} \frac{\partial T}{\partial t}=k \nabla^{2} T+R \varepsilon \frac{\partial m}{\partial t} .
\end{gathered}
$$

Equations (2.9) and (2.10) have another complication: the value of $\varepsilon$ changes from 0 to 1 depending on the significance of liquid transfer relative to the vapour diffusion within the material and, in turn, depending on the nature of the material. We expect it to increase with temperature. The effect of the phase change coefficient on the moisture and temperature regimes can be explored using the model given by (2.9) and (2.10).

\section{Analytical solutions}

Equations (2.9) and (2.10) form a pair of coupled nonhomogenous second-order PDEs. The applicability of general methods for solving coupled PDEs is limited by properties of 
the region over which the problem is defined, as well as by boundary and initial conditions. For simplicity, we reduce the problem to a single spatial dimension and substitute $\alpha=k / \rho_{0} c$ and $\beta=R / \rho_{0} c$ :

$$
\begin{aligned}
\frac{\partial}{\partial t} m(x, t) & =a_{m} \frac{\partial^{2}}{\partial x^{2}} m(x, t)+a_{m} \delta \frac{\partial^{2}}{\partial x^{2}} T(x, t), \\
\frac{\partial}{\partial t} T(x, t) & =\alpha \frac{\partial^{2}}{\partial x^{2}} T(x, t)+\varepsilon \beta \frac{\partial}{\partial t} m(x, t), \quad 0<x<l,
\end{aligned}
$$

with initial and boundary conditions defining the problem over the region $[0, l]$ along the $x$ axis:

$$
\begin{array}{ll}
m(x, 0)=m_{i}(x)=M_{i}, & T(x, 0)=T_{i}(x)=T_{i}, \\
m(0, t)=m(l, t)=M_{\infty}, & T(0, t)=T(l, t)=T_{\infty} .
\end{array}
$$

One of the widely accepted approaches for solving systems of PDEs is applying integral transforms to reduce the problem to simple differential equations. We found that Laplace and Fourier transforms in the time domain were applicable, but did not significantly simplify the problem. Transformations applied in the spatial domain would have done so, but boundary conditions ruled out both Fourier and Laplace transforms. Fourier transform did not prove viable because of the finite nature of the spatial domain, while Laplace transform could not be utilised due to the lack of information on the derivatives of the solutions at the boundaries. Efforts made using transforms in the spatial domain thus yielded results satisfying the PDEs, but inconsistent with the boundary and/or initial conditions.

The method finally used to obtain satisfactory results is based on solving the nonhomogenous heat conduction problem by means of eigenvalues and eigenfunctions [1]. The steps for solving the partial differential equation

$$
\frac{\partial F(x, t)}{\partial t}=\gamma \frac{\partial^{2} F(x, t)}{\partial x^{2}}+D(x, t)
$$

for $F(x, t)$ with appropriate initial and boundary conditions, is as follows ( $\gamma$ is a constant, and $D(x, t)$ is the "disturbing" nonhomogenous term):

(1) solve the appropriate homogenous problem (assuming $D(x, t)=0$ ) to find the eigenvalues and eigenfunctions needed to construct the solution as an infinite sum;

(2) express all functional terms in the nonhomogenous problem in terms of the obtained eigen functions;

(3) solve the resulting equation of infinite sums for the coefficients needed to construct the solution $F(x, t)$ by exploiting properties of eigenvalues and eigenfunctions. 
To apply this method to the original problem of solving a coupled pair of equations, the coupling terms in each equation are treated as the nonhomogenous "disturbing" terms $(D(x, t)$ s). It is assumed that the solutions for $m(x, t)$ and $T(x, t)$ in $(3.1)$ can be expressed using infinite sums:

$$
\begin{aligned}
& m(x, t)=C+\sum_{n=1}^{\infty} a_{n}(t) \Phi_{n}(x), \\
& T(x, t)=D+\sum_{n=1}^{\infty} b_{n}(t) \Psi_{n}(x),
\end{aligned}
$$

where $\Phi_{n}(x)$ and $\Psi_{n}(x)$ are eigenfunctions independent of time, coefficients $a_{n}(t)$ and $b_{n}(t)$ are functions of time only, and $C$ and $D$ are translating functions derived from the initial and boundary conditions. In the special case of (3.2) and (3.3), where boundary conditions are symmetric (and constant) and the initial functions are constants, $C$ and $D$ are constants.

The appropriate homogenous problem to (3.1) is

$$
\begin{aligned}
\frac{\partial}{\partial t} m_{H}(x, t) & =a_{m} \frac{\partial^{2}}{\partial x^{2}} m_{H}(x, t), \\
\frac{\partial}{\partial t} T_{H}(x, t) & =\alpha \frac{\partial^{2}}{\partial x^{2}} T_{H}(x, t) .
\end{aligned}
$$

Note that the equations in (3.6) are no longer coupled. Also note that both have the same general form

$$
\frac{\partial}{\partial t} f(x, t)=\sigma \frac{\partial^{2}}{\partial x^{2}} f(x, t)
$$

The assumption can be made that $m_{H}(x, t)$ and $T_{H}(x, t)$ have identical eigenvalues and eigenfunctions. Note that homogenous PDEs with the above form can be solved using the method of separation of variables. Doing so suggests that the eigenvalues and eigenfunctions for the corresponding boundary conditions (3.3) are

$$
\lambda_{n}=\frac{n^{2} \pi^{2}}{l^{2}}, \quad \Psi_{n}(x)=\Phi_{n}(x)=\sin (\sqrt{\lambda} x)=\sin \left(\frac{n \pi}{l} x\right), \quad n=1,2, \ldots, \infty .
$$

From (3.5a) and (3.5b) we thus derive

$$
\begin{gathered}
m_{n}(x, t)=a_{n}(t) \Phi_{n}(x), \\
T_{n}(x, t)=b_{n}(t) \Phi_{n}(x) .
\end{gathered}
$$

Because differential operators are linear, $m_{n}(x, t)$ and $T_{n}(x, t)$ must satisfy the equations in (3.1) for all $n$. Substituting (3.9) into (3.1),

$$
\begin{aligned}
& \sum_{n} a_{n}^{\prime}(t) \Phi_{n}(x)=a_{m} \sum_{n} a_{n}(t) \Phi_{n}^{\prime \prime}(x)+a_{m} \delta \sum_{n} b_{n}(t) \Phi_{n}^{\prime \prime}(x), \\
& \sum_{n} b_{n}^{\prime}(t) \Phi_{n}(x)=\alpha \sum_{n} b_{n}(t) \Phi_{n}^{\prime \prime}(x)+\beta \varepsilon \sum_{n} a_{n}^{\prime}(t) \Phi_{n}(x) .
\end{aligned}
$$


Substituting the identity that is easy to verify,

$$
\Phi_{n}^{\prime \prime}(x)=-\lambda_{n} \Phi_{n}(x)
$$

and rearranging to one summation the equations become

$$
\begin{gathered}
\sum_{n}\left[a_{n}^{\prime}(t)+a_{m} \lambda_{n} a_{n}(t)+a_{m} \delta \lambda_{n} b_{n}(t)\right] \Phi_{n}(x)=0, \\
\sum_{n}\left[b_{n}^{\prime}(t)+\alpha \lambda_{n} b_{n}(t)-\beta \varepsilon a_{n}^{\prime}(t)\right] \Phi_{n}(x)=0 .
\end{gathered}
$$

The equations in (3.12) can only hold for all $0<x<l$ if the quantities in the square brackets are 0 for all $n$ because the eigenfunctions form an orthogonal set. Thus to find $a_{n}$ and $b_{n}$, we must solve the coupled pair of first-order differential equations for each value of $n$ :

$$
\begin{array}{r}
a_{n}^{\prime}(t)+a_{m} \lambda_{n} a_{n}(t)+a_{m} \delta \lambda_{n} b_{n}(t)=0, \\
b_{n}^{\prime}(t)+\alpha \lambda_{n} b_{n}(t)-\beta \varepsilon a_{n}^{\prime}(t)=0 .
\end{array}
$$

Initial values for $a_{n}(t)$ and $b_{n}(t)$ are derived from the original initial and boundary conditions (3.2) and (3.3) through a translation [14]:

$$
\begin{aligned}
a_{n}(0) & =A_{n}=\frac{2}{l} \int_{x=0}^{l}\left(m_{i}(x)-M_{\infty}\right) \Phi_{n}(x) d x \\
& =\frac{-2\left(M_{i}-M_{\infty}\right)}{n \pi}[\cos (n \pi)-1], \\
b_{n}(0) & =B_{n}=\frac{2}{l} \int_{x=0}^{l}\left(T_{i}(x)-T_{\infty}\right) \Phi_{n}(x) d x \\
& =\frac{-2\left(T_{i}-T_{\infty}\right)}{n \pi}[\cos (n \pi)-1] .
\end{aligned}
$$

$A_{n}$ and $B_{n}$ are actually the coefficients used to express the original initial functions as infinite linear combinations of $\Phi_{n}(x)$. Note that the cosine term is 1 if $n$ is even and -1 if $n$ is odd. This cancels all harmonics of $\Phi(x)$ that are nonsymmetric on the interval $0<x<l$. To solve (3.13) the Laplace transform in time $(f(t) \rightarrow F(s))$ is applied; simplifying the differential equations to algebraic equations (omitting the indices $n$ ),

$$
\begin{gathered}
s A-A+a_{m} \lambda A+a_{m} \delta \lambda B=0, \\
s B-B+\alpha \lambda B-\beta \varepsilon(s A-A)=0 .
\end{gathered}
$$


Solving for $A$ and $B$ and applying the inverse Laplace transformation to the results we obtain the solutions for $a_{n}(t)$ and $b_{n}(t)$ :

$$
\begin{aligned}
a_{n}(t) & =\frac{p_{1} A_{n}+C_{n}}{p_{1}-p_{2}} \exp \left(p_{1} t\right)+\frac{p_{2} A_{n}+C_{n}}{p_{2}-p_{1}} \exp \left(p_{2} t\right), \\
C_{n} & =-B_{n} a_{m} \lambda_{n} \delta+\alpha \lambda_{n} A_{n}+\beta \varepsilon A_{n} a_{m} \lambda_{n} \delta, \\
b_{n}(t) & =\frac{p_{1} B_{n}+D_{n}}{p_{1}-p_{2}} \exp \left(p_{1} t\right)+\frac{p_{2} B_{n}-D_{n}}{p_{2}-p_{1}} \exp \left(p_{2} t\right), \\
D_{n} & =B_{n} a_{m} \lambda_{n}-\beta A_{n} a_{m} \lambda_{n} \varepsilon,
\end{aligned}
$$

where

$$
\begin{aligned}
& p_{1}=-\frac{1}{2}\left(\beta \varepsilon a_{m} \delta+\alpha+a_{m}-\sqrt{\left(\beta \varepsilon a_{m} \delta\right)^{2}+2 \beta \varepsilon \delta a_{m}^{2}+2 \beta \varepsilon \delta a_{m} \alpha+a_{m}^{2}+\alpha^{2}-2 a_{m} \alpha}\right) \\
& p_{2}=-\frac{1}{2}\left(\beta \varepsilon a_{m} \delta+\alpha+a_{m}+\sqrt{\left(\beta \varepsilon a_{m} \delta\right)^{2}+2 \beta \varepsilon \delta a_{m}^{2}+2 \beta \varepsilon \delta a_{m} \alpha+a_{m}^{2}+\alpha^{2}-2 a_{m} \alpha}\right) .
\end{aligned}
$$

(Note that for positive constants poles $p_{1}$ and $p_{2}$ are always negative, and the singularity at $p_{1}=p_{2}$ is bounded.) Finally, the solution for $m(x, t)$ and $T(x, t)$ consistent with the "initial and boundary conditions" can be expressed as

$$
\begin{aligned}
& m(x, t)=M_{\infty}+\sum_{n=1}^{\infty} a_{n}(t) \Phi_{n}(x), \\
& T(x, t)=T_{\infty}+\sum_{n=1}^{\infty} b_{n}(t) \Phi_{n}(x) .
\end{aligned}
$$

(Note that since (3.14) was a solution for the translated initial values, we compensate by defining $C$ and $D$ from (3.5) accordingly.)

The results have also been verified by substituting zeros for coupling parameters and comparing results with those obtained from solving the noncoupled homogenous pair of equations $\left(m_{H}(x, t), T_{H}(x, t)\right)$.

\section{Exploration of analytical solutions}

Equations (3.19) provide analytical solutions to the PDEs that describe simultaneous moisture and heat transfers within a porous body, and the solutions determine the temperature and moisture profiles. It is important to obtain insight into the behaviour of 


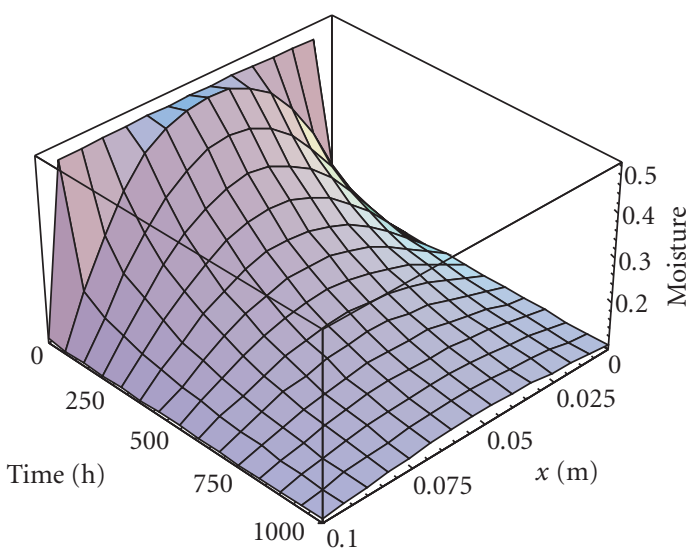

(a)

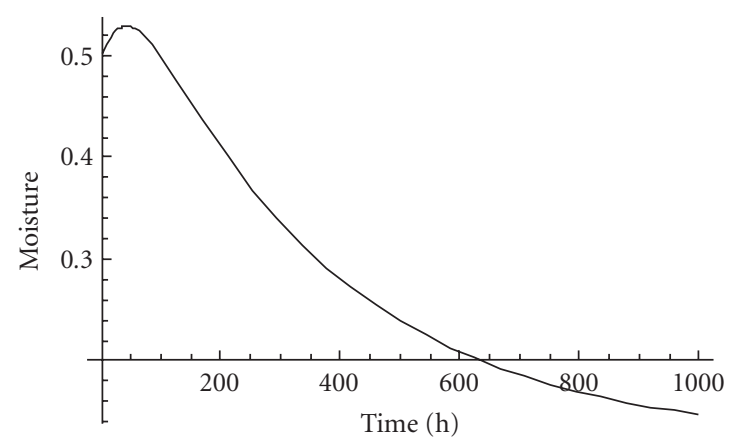

(b)

Figure 4.1. (a) A 3D moisture content profile for $l=0.1 \mathrm{~m}$. (b) Moisture content at $x=0.05 \mathrm{~m}$ versus time for $l=0.1 \mathrm{~m}$.

the solutions with respect to the coupling parameters ( $\delta$ and $\varepsilon$ ) as well as material properties, for example, to know the conditions under which the moisture and temperature coupling is important. The reduced dimensionality of the problem makes it easy to understand the interactions among various factors without unduly complicating the study.

We assume the following numerical values for the drying of an infinite "panel" of $P$. radiata $0.10 \mathrm{~m}$ thick, with drying temperature and humidity held constant along both of its infinite faces: $R=2400 \mathrm{~kJ} / \mathrm{kg}, a_{m}=3.0 \times 10^{-6} \mathrm{~m}^{2} / \mathrm{h}, \rho_{0}=500 \mathrm{~kg} / \mathrm{m}^{3}, c=1284 \mathrm{~J} / \mathrm{kg}^{\circ} \mathrm{C}$, $k=0.12 \mathrm{~W} / \mathrm{m} \mathrm{K}, T_{i}=10^{\circ} \mathrm{C}, T_{\infty}=80^{\circ} \mathrm{C}, M_{i}=0.5$ (dry basis), and $M_{\infty}=0.12$ (dry basis). After experimenting with the number of terms in the solutions to obtain approximate solutions, we found that, for the parameter regimes tested, 20 terms would be sufficient to obtain convergences to consistent values having differences between successive 


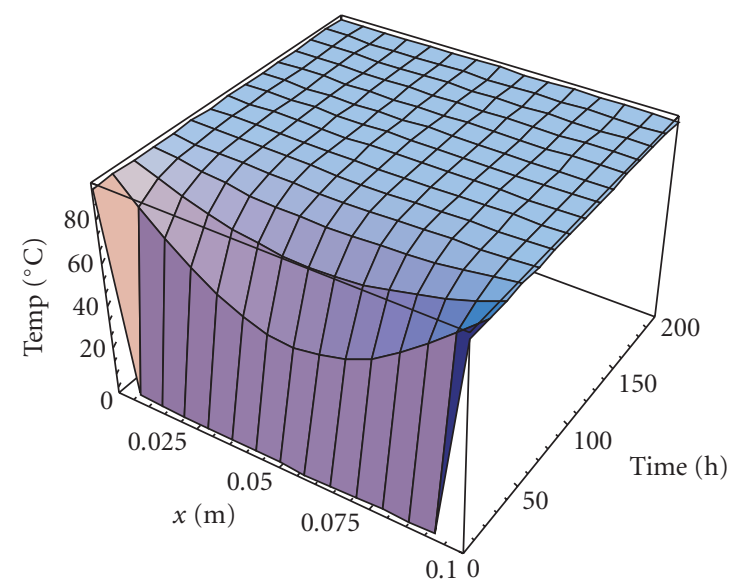

(a)

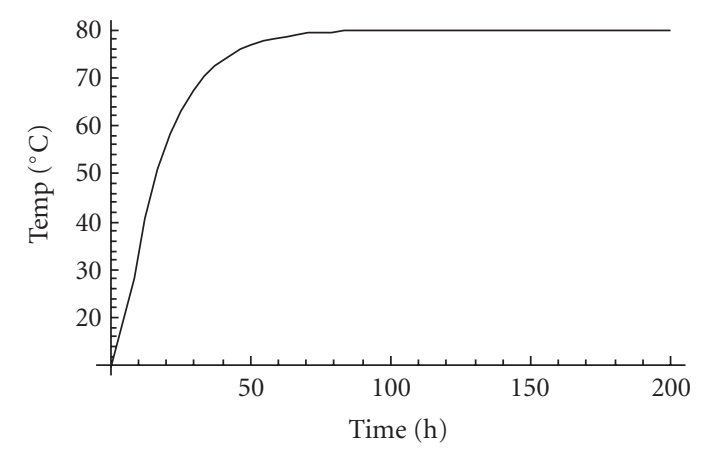

(b)

Figure 4.2. (a) A 3D temperature profile for $l=0.1 \mathrm{~m}$. (b) Temperature at $x=0.05 \mathrm{~m}$ versus time for $l=0.1 \mathrm{~m}$.

summations of less than $0.001^{\circ} \mathrm{C}$ and 0.001 (decimal, dry basis) for temperatures and moisture contents, respectively. Figures 4.1 and 4.2 show the moisture and temperature profiles, respectively, by assuming the values $\delta=0.01^{\circ} \mathrm{C}^{-1}$, and $\varepsilon=0.1$ for the coupling parameters. The moisture and temperature versus time at $x=0.05 \mathrm{~m}$ are given in Figures $4.1 \mathrm{~b}$ and $4.2 \mathrm{~b}$, respectively. While the rise in temperature is sharp, the decrease in moisture is slow because of the relatively low value of the total diffusion coefficient $a_{m}$, and the thermal diffusivity $\alpha$ is relatively high. We can expect the coupling effects to be negligible when the temperature gradients are very small after initial hours of drying. Moisture is increased slightly at the beginning because of the moisture transfer driven by the temperature gradient. 


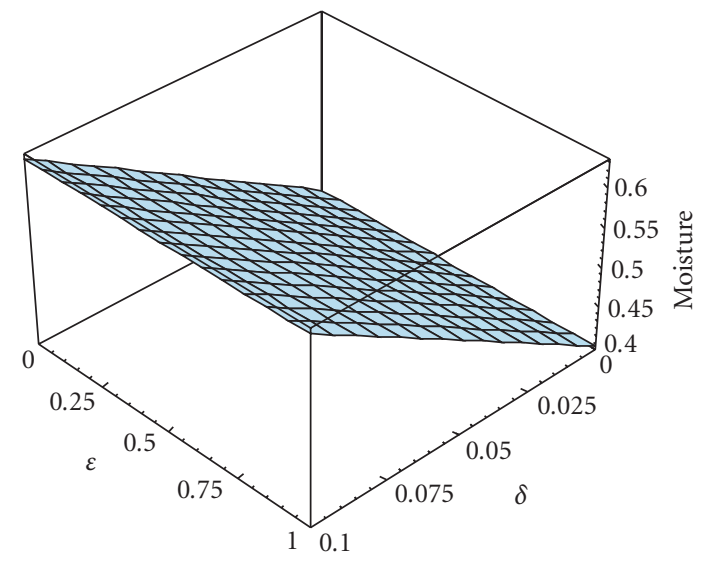

Figure 4.3. Moisture variation with the thermo-gradient coefficient $(\delta)$ and the phase change coefficient $(\varepsilon)$ for $x=0.05 \mathrm{~m}, t=200$ hours, and $l=0.1 \mathrm{~m}$.

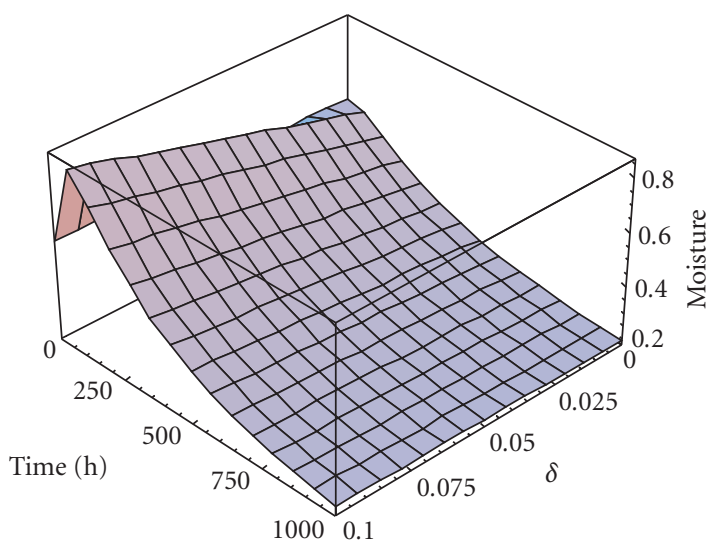

Figure 4.4. The transient moisture content versus the thermo-gradient coefficient and time for $x=$ $0.05 \mathrm{~m}, l=0.1 \mathrm{~m}$, and $\varepsilon=0.1$.

Figure 4.3 shows the variation of the moisture content at $x=0.05 \mathrm{~m}, t=200$ hours with $\delta$ and $\varepsilon$ for the same set of values given above. While the moisture is insensitive to the changes of $\varepsilon$, the thermo-gradient coefficient $\delta$ has a significant effect on moisture. For a given total diffusion coefficient $a_{m}, \delta$ indicates the significance of moisture transfer due to temperature gradient. Therefore, within realistic limits, $\delta$ can be expected to influence drying as seen in the transient moisture profiles at the same location as before (Figure 4.4). For P. radiata, the nature of thermodiffusion effect has an impact on the moisture content of the material during the first 300 hours.

To illustrate how the thickness of the panel affects the moisture and temperature profiles, we have produced Figures 4.5, 4.6, and 4.7 for a thickness ( $l$ ) of $0.5 \mathrm{~m}$. Temperature 


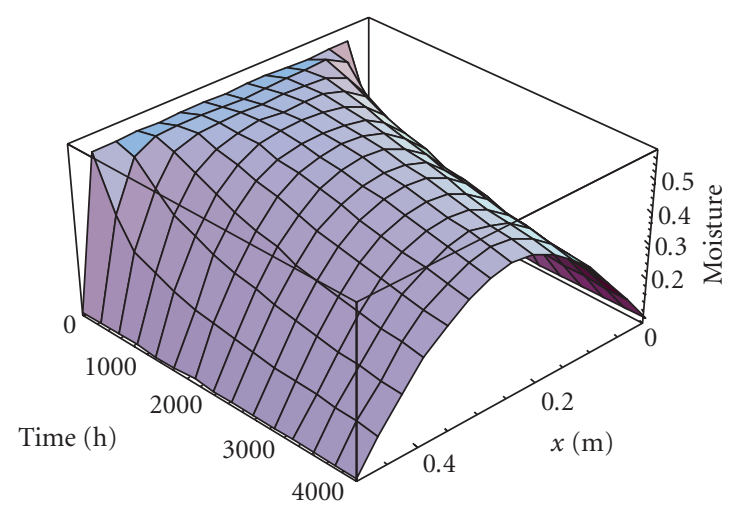

(a)

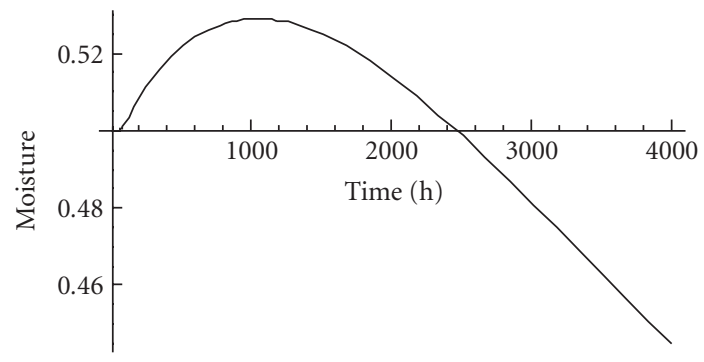

(b)

Figure 4.5. (a) A 3D moisture content profile for $l=0.5 \mathrm{~m}$. (b) Moisture content at $x=0.25 \mathrm{~m}$ versus time for $l=0.5 \mathrm{~m}$.

at $x=0.25$ (mid point) takes almost 1500 hours to reach close to the outside temperature when $l=0.5$ (Figure 4.6b), whereas the mid-point temperature reaches the outside temperature within 70 hours when $l=0.1$ (Figure 4.2b). The moisture profile at $x=0.25 \mathrm{~m}$ is slow to change (Figure 4.2) as compared to Figure 4.1. The thermo-gradient coefficient $(\delta)$ is significant for a longer period of time (Figures 4.7 and 4.8), and therefore it can be argued that the drying models purely based on moisture potential gradients without taking into account dynamic changes in temperature would not give realistic results when the thickness is large (greater than $0.2 \mathrm{~m}$ ).

It was found that the values of $\alpha$ and $a_{m}$ should be in realistic ranges for moisture profiles to have meaningful values. This is illustrated in Figure 4.9 where the moisture content at $x=0.25 \mathrm{~m}, t=500$ hours is plotted against $\alpha$ and $a_{m}$ for $\varepsilon=0.1$ and $\delta=0.1$. The regimes of $\alpha$ and $\beta$ which would produce realistic moisture contents at $x=0.25 \mathrm{~m}$, $t=500$ hours are given in Figure 4.10, and it is seen that low $\beta$ (i.e., the density and/or specific heat of the material is high) has a significant effect on the moisture profile at small values of $\alpha$ and vice versa. 


\section{On modelling the drying of porous materials}

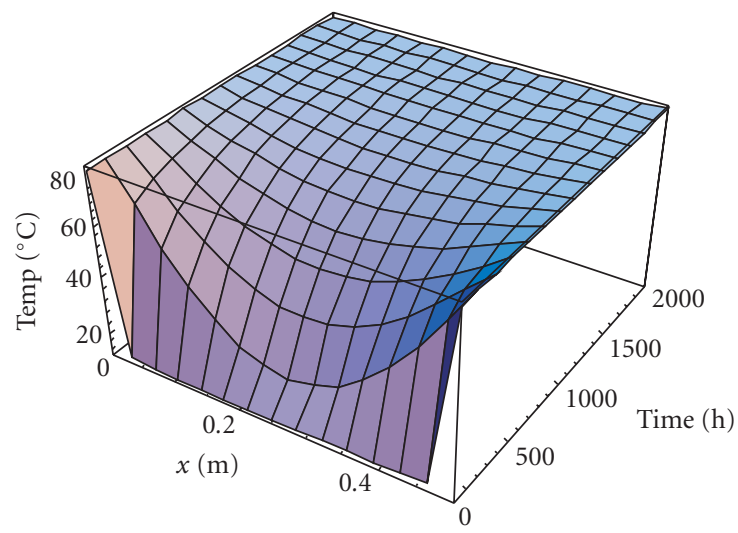

(a)

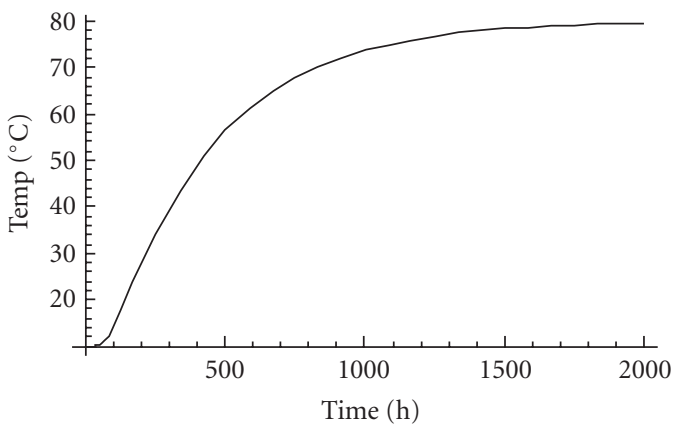

(b)

Figure 4.6. (a) A 3D temperature profile for $l=0.5 \mathrm{~m}$. (b) Temperature at $x=0.05 \mathrm{~m}$ versus time for $l=0.5 \mathrm{~m}$.

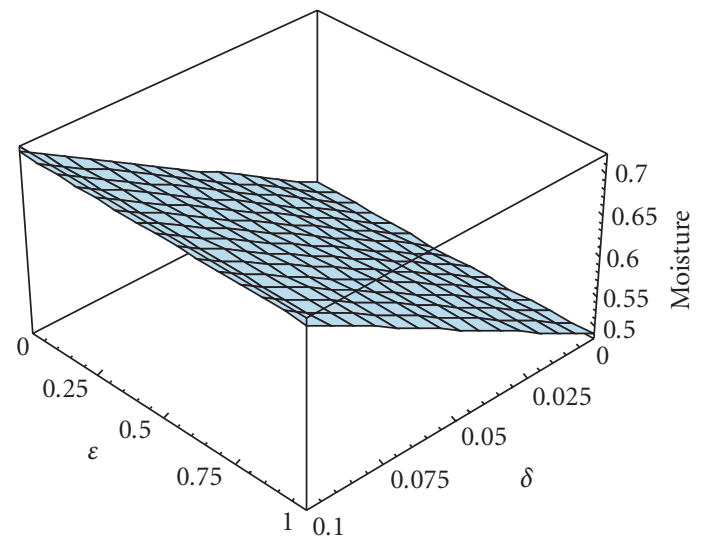

Figure 4.7. Moisture variation with the thermo-gradient coefficient $(\delta)$ and the phase change coefficient $(\varepsilon)$ for $x=0.25 \mathrm{~m}, t=500$ hours, and $l=0.5 \mathrm{~m}$. 


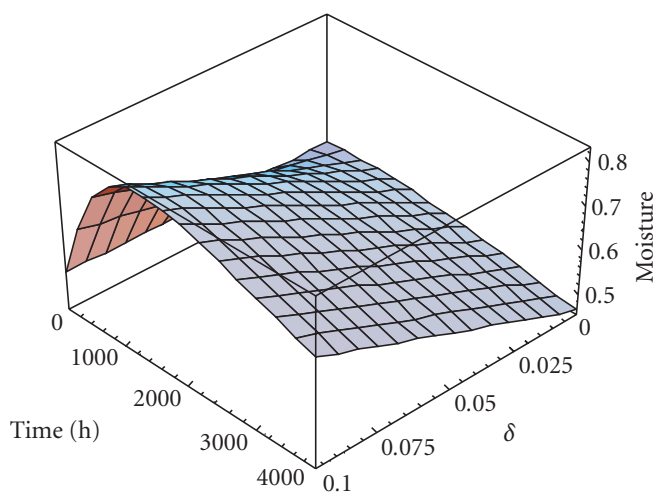

Figure 4.8. The transient moisture content versus the thermo-gradient coefficient and time for $x=$ $0.25 \mathrm{~m}, l=0.5 \mathrm{~m}$, and $\varepsilon=0.1$.

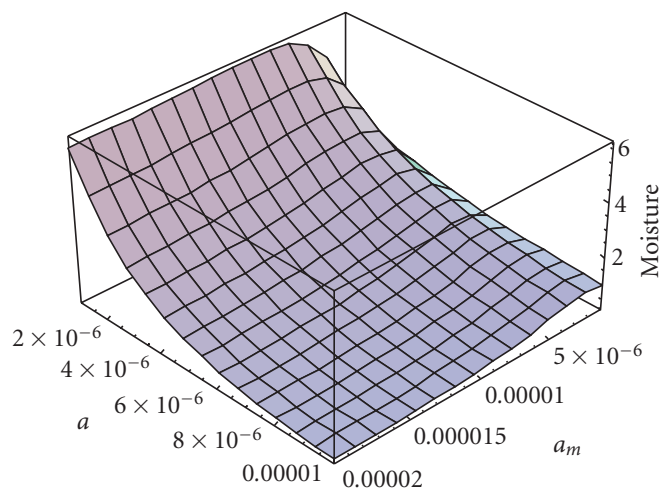

Figure 4.9. Moisture as a function of $a_{m}$ and $\alpha$ for $x=0.25 \mathrm{~m}, t=500$ hours, and $l=0.5 \mathrm{~m}$.

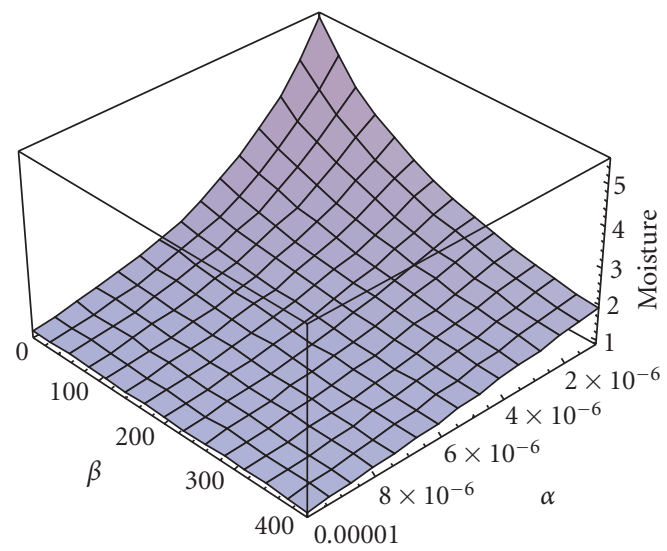

Figure 4.10. Moisture at $x=0.25 \mathrm{~m}, t=500$ hours as a function of $\alpha$ and $\beta$ for $\varepsilon=0.1, \delta=0.1$, and $l=0.5 \mathrm{~m}$. 


\section{Conclusion}

Coupled PDEs that govern the drying of porous materials have been solved analytically, and the solutions were explored to show that thermodiffusion effects cannot be ignored when drying moist porous materials is concerned. The thermo gradient coefficient is a significant coupling parameter whereas the influence of the phase change coefficient has lesser significance in the dynamics of heat and moisture profiles. Therefore, vapour diffusion relative to liquid transfer can be ignored in drying such materials as $P$. radiata. However, this should be explored for each material relevant boundary and initial conditions using the analytical solutions. The solutions enable us to experimentally determine the coupling parameters in a setting where both the moisture and heat transfers are important without resorting to a simplified set of equations that govern heat and mass transfer separately.

\section{Acknowledgments}

Financial assistance given by the Foundation for Research, Science and Technology (FoRST), New Zealand, for this work is appreciated. Assistance from Jozseph Koloszar, a visiting student from Hungary, is gratefully acknowledged.

\section{References}

[1] W. E. Boyce and R. C. DiPrima, Elementary Differential Equations and Boundary Value Problems, 3rd ed., John Wiley \& Sons, New York, 1977.

[2] H. S. Carslaw and J. C. Jaeger, Conduction of Heat in Solids, Oxford Science Publications, The Clarendon Press; Oxford University Press, New York, 1990.

[3] M. S. Chinnan and J. H. Young, A study of diffusion equations describing moisture movement in peanut pods. I: Comparison of vapor and liquid diffusion equations, Trans. ASAE. 20 (1977), no. 3, 539-546.

[4] J. Crank, The Mathematics of Diffusion, Oxford Science Publication, Oxford University Press, Oxford, 1990.

[5] A. A. Dolinskij, A. Sh. Dorfman, and B. V. Davydenko, Conjugate heat and mass transfer in continuous processes of convective drying, Int. J. Heat Mass Transfer 34 (1991), no. 11, 28832889.

[6] G. D. Fulford, A survey of recent Soviet research on the drying of solids, Can. J. Chem. Eng. 47 (1969), 378-391.

[7] D. Kulasiri, Simulation of deep-bed drying of Virginia peanuts to minimize energy use, Ph.D. thesis, Virginia Polytechnic Institute and State University, Blacksburg, 1990.

[8] G. D. Kulasiri and S. Samarasinghe, Modelling heat and mass transfer in drying of biological materials: a simplified approach for materials with small dimensions, Ecological Modelling 86 (1996), 163-167.

[9] A. V. Luikov, Heat and Mass Transfer in Capillary-Porous Bodies, Pergamon Press, 1966.

[10] K. Murugesan, H. N. Suresh, K. N. Seetharamu, P. A. A. Narayana, and T. Sundararajan, A theoretical model of brick drying as a conjugate problem, Int. J. Heat Mass Transfer 44 (2001), no. 21, 4075-4086.

[11] L. S. Olivera and K. Haghighi, Conjugate heat and mass transfer in convective drying of porous media, Numr. Heat Transfer 34 (1998), no. 2, 105-117.

[12] J. N. Reddy, An Introduction to the Finite Element Method, 2nd ed., McGraw-Hill, New York, 1993. 
[13] A. Z. Sahin and I. Dincer, Graphical determination of drying process and moisture transfer parameters for solids drying, Int. J. Heat Mass Transfer 45 (2002), no. 16, 3267-3273.

[14] H. R. Thomas, K. Morgan, and R. W. Lewis, A fully nonlinear analysis of heat and mass transfer problems in porous bodies, Int. J. Numer. Methods Eng. 15 (1980), no. 9, 1381-1393.

[15] J. M. Troeger and J. L. Butler, Simulation of solar peanut drying, Trans. ASAE. 22 (1979), no. 4, 906-911.

Don Kulasiri: Centre for Advanced Computational Solutions (C-fACS), Lincoln University, P.O. Box 84, Canterbury, New Zealand

E-mail address: kulasird@lincoln.ac.nz

Ian Woodhead: Lincoln Technology, Lincoln Ventures Ltd, Lincoln University, Canterbury, New Zealand

E-mail address: woodhead@lincoln.ac.nz 


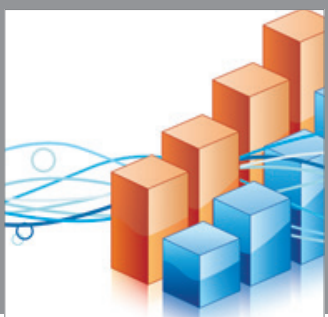

Advances in

Operations Research

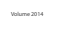

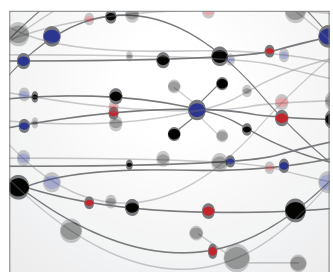

\section{The Scientific} World Journal
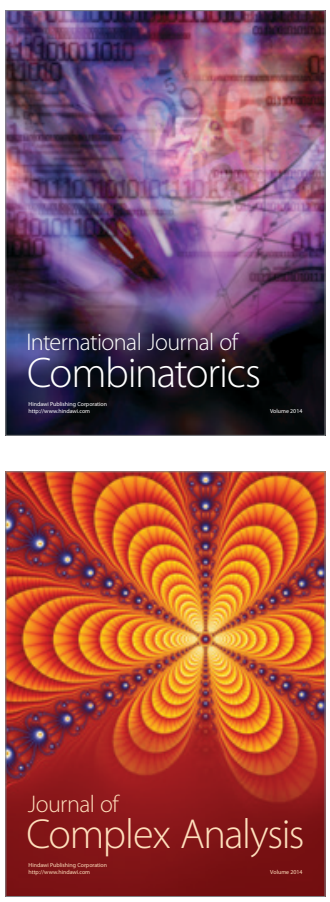

International Journal of

Mathematics and

Mathematical

Sciences
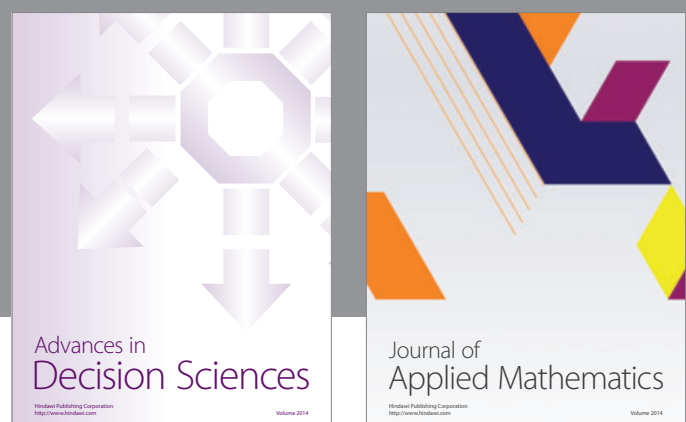

Journal of

Applied Mathematics
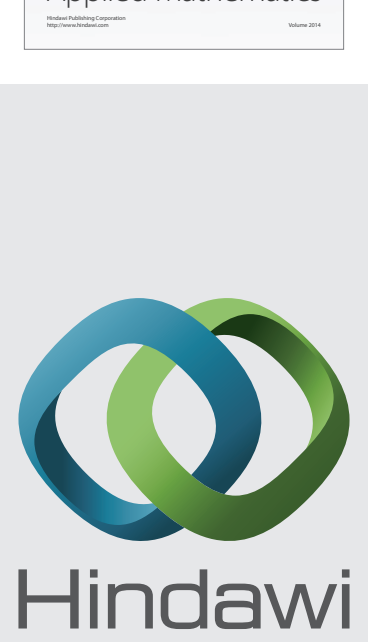

Submit your manuscripts at http://www.hindawi.com
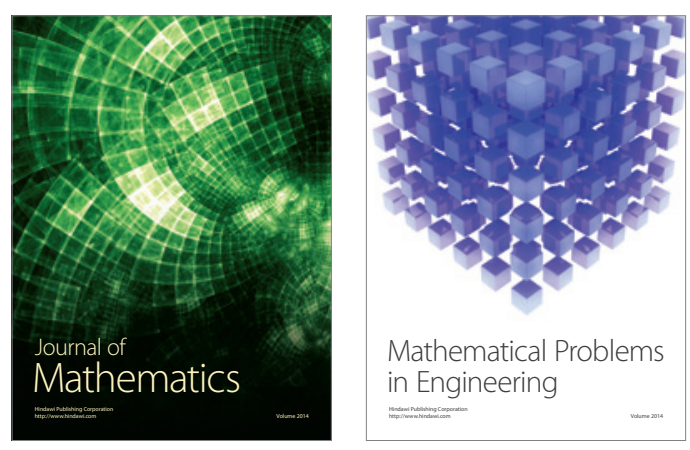

Mathematical Problems in Engineering
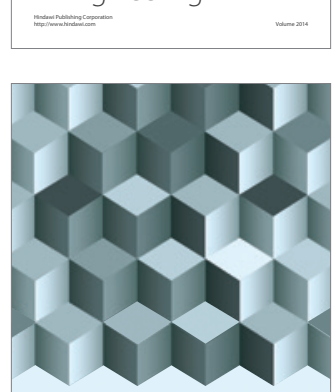

Journal of

Function Spaces
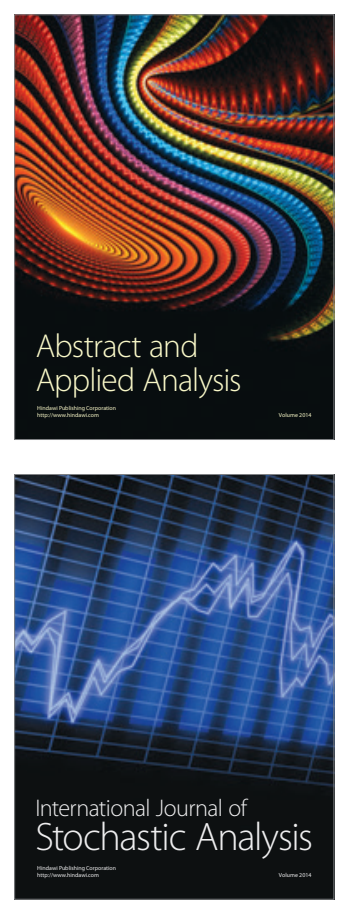

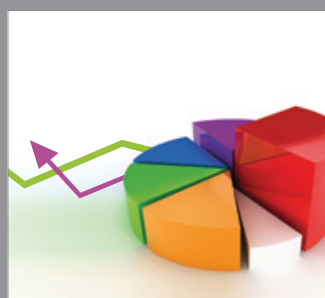

ournal of

Probability and Statistics

Promensencen
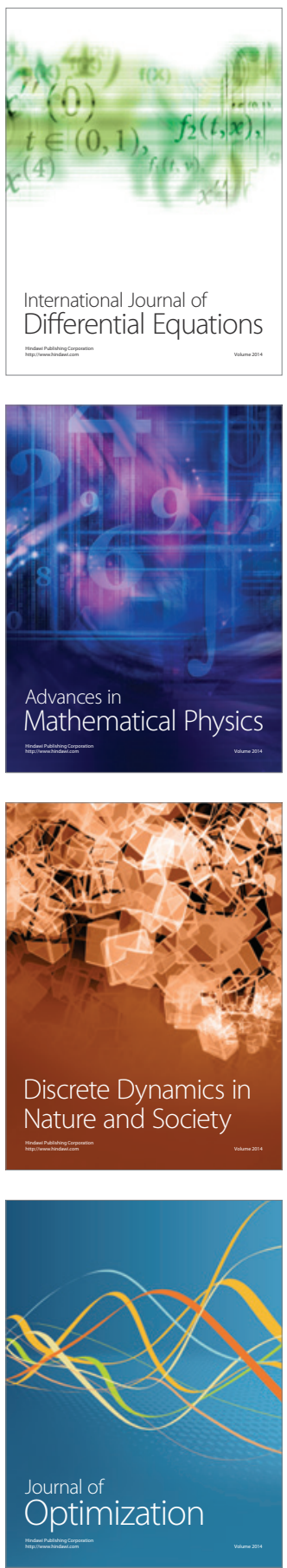